\title{
Robustness Analysis in Sequential Statistical Decisions
}

\author{
Alexey Kharin \\ Belarusian State University
}

\begin{abstract}
The sequential statistical decision making is considered. Performance characteristics (error probabilities and expected sample sizes) for the sequential statistical decision rules (tests) are analysed. Both cases of simple and composite hypotheses are considered. Asymptotic expansions under distortions are constructed for the performance characteristics enabling robust sequential test construction.
\end{abstract}

Keywords: sequential statistical decision, test, hypothesis, distortion, robustness.

\section{Introduction}

In many fields of statistical methodology applications, especially in medicine, finance, quality control, problems of statistical decision making are topical (Ghosh and Sen 1991), (Jennison and Turnbull 2000). In statistical decision making, one of the most important problems is to construct decisions providing the requested accuracy on the basis of the minimal information (minimal number of observations). To solve this problem, the sequential approach (Wald 1947), (Lai 2001) is used. Within this approach, the number of observations required to provide the prescribed performance is not fixed a priori, but is considered as a random variable that depends on random observations. To find the optimal dependency is a complicated task, but this scheme of decision making requires a number of observations that is essentially less than what is required by the approach based on fixed sample sizes (see Aivazian (1959)).

The assumed hypothetical probability models used in the sequential approach are quite often distorted in practice (Huber and Ronchetti 2009), (Maevskii and Kharin 2002), (Kharin 2011), (Kharin 2005). Therefore, the problem of robustness analysis (Kharin and Zhuk 1998), (Kharin 1997), (Kharin and Vecherko 2013), (Galinskij and Kharin 1999) for sequential statistical decision making under distortions is an important one.

In (Quang 1985) the robustness analysis is given for a special hypothetical model in case of simple hypotheses. An empirical study of robustness is performed in (Pandit and Gudaganavar 2009) for the scale parameter of gamma and exponential distributions. Here we systemize some of the results developed by the author for quantitative robustness analysis of sequential decision rules. 


\section{Case of simple hypotheses}

Let on a probability space $(\Omega, \mathcal{F}, \mathcal{P})$ random variables $x_{t} \in U=\left\{u_{1}, u_{2}, \ldots, u_{M}\right\}$ be observed, $t \in \mathbf{N}$, independent in total and identically distributed. The probability distribution of each random variable depends on parameter value $\theta \in \Theta=\left\{\theta_{0}, \theta_{1}\right\}, \theta_{0}, \theta_{1} \in \mathbf{R}, \theta_{0} \neq \theta_{1}$, and satisfy the grid condition:

$$
P(u ; \theta)=P_{\theta}\left\{x_{t}=u\right\}=a^{-J(u ; \theta)}, t \in \mathbf{N}, u \in U,
$$

where $a \in \mathbf{Q}, a>1 ; J(u ; \theta): U \times \Theta \longrightarrow \mathbf{Z}_{+}$is a function, satisfying the normalization condition

$$
\sum_{u \in U} a^{-J(u ; \theta)}=1
$$

Concerning the parameter value $\theta$ of the probability distribution (1) the following two simple hypotheses are considered:

$$
\mathcal{H}_{0}: \theta=\theta_{0}, \mathcal{H}_{1}: \theta=\theta_{1}
$$

Denote the statistic

$$
\Lambda_{n}=\Lambda_{n}\left(x_{1}, x_{2}, \ldots, x_{n}\right)=\sum_{t=1}^{n} \lambda_{t}
$$

where $\lambda_{t}=\log _{a}\left(P\left(x_{t} ; \theta_{1}\right) / P\left(x_{t} ; \theta_{0}\right)\right)=J\left(x_{t} ; \theta_{0}\right)-J\left(x_{t} ; \theta_{1}\right) \in \mathbf{Z}$ is the logarithm of the likelihood ratio, calculated by the observation $x_{t}$.

In the sequential probability ratio test (SPRT, Wald test) for hypotheses (3) testing after $n$ observations $(n=1,2, \ldots)$ the decision is made according to:

$$
d=\mathbf{1}_{\left[C_{+},+\infty\right)}\left(\Lambda_{n}\right)+2 \cdot \mathbf{1}_{\left(C_{-}, C_{+}\right)}\left(\Lambda_{n}\right) .
$$

The values $d=0$ and $d=1$ correspond to stopping of the observation process and acceptance of the hypotheis $\mathcal{H}_{0}$ (if $d=0$ ) or $\mathcal{H}_{1}$ (if $d=1$ ) by $n$ observations. If $d=2$, the observation number $(n+1)$ should be made. In (5) parameters $C_{-}, C_{+} \in \mathbf{Z}$ are calculated as follows:

$$
C_{-}=\left[\log _{a}\left(\beta_{0} /\left(1-\alpha_{0}\right)\right)\right], C_{+}=\left[\log _{a}\left(\left(1-\beta_{0}\right) / \alpha_{0}\right)\right]
$$

where $\alpha_{0}, \beta_{0}$ are the requested values of the error type I and II probabilities respectively; [·] means the integer part of an argument.

Let the model described above is distorted - instead of (1) observations $x_{1}, x_{2}, \ldots$ are obtained from the mixture of discrete distributions:

$$
\bar{P}(u ; \theta)=\bar{P}_{\theta}\left\{x_{t}=u\right\}=(1-\varepsilon) P(u ; \theta)+\varepsilon \tilde{P}(u ; \theta), t \in \mathbf{N}, u \in U,
$$

where $\varepsilon \in\left[0, \frac{1}{2}\right)$ is the "contamination" probability; the "contaminating" probability distribution has the form

$$
\tilde{P}(u ; \theta)=a^{-\tilde{J}(u ; \theta)}, u \in U, \theta \in \Theta,
$$

where $\tilde{J}(u ; \theta): U \times \Theta \longrightarrow \mathbf{Z}_{+}$is a function, different from $J(\cdot)$, and satisfying $\sum_{u \in U} a^{-\tilde{J}(u ; \theta)}=$ 1.

For $i \in\left(C_{-}, C_{+}\right)$denote

$$
\begin{aligned}
\tilde{p}_{i j}^{(k)}(g)= \begin{cases}\sum_{u \in U} \delta_{g\left(J\left(u, \theta_{0}\right)-J\left(u ; \theta_{1}\right)\right), j-i} \tilde{P}\left(u ; \theta_{k}\right), & j \in\left(C_{-}, C_{+}\right), \\
\sum_{u \in U} \mathbf{1}_{\left(-\infty, C_{-}\right]}\left(g \left(J\left(u, \theta_{0}\right)-\right.\right. & j=C_{-}, \\
\left.\left.-J\left(u, \theta_{1}\right)\right)+i\right) \tilde{P}\left(u ; \theta_{k}\right), & j=C_{+} ; \\
\sum_{u \in U} \mathbf{1}_{\left[C_{+},+\infty\right)}\left(g \left(J\left(u, \theta_{0}\right)-\right.\right. & \tilde{P}\left(u ; \theta_{k}\right), \\
\left.\left.-J\left(u, \theta_{1}\right)\right)+i\right) & \tilde{P}^{(k)}(g)=\left(\tilde{p}_{i j}^{(k)}(g)\right), \tilde{\pi}^{(k)}(g)=\left(\tilde{\pi}_{i}^{(k)}(g)\right), i \in\left(C_{-}, C_{+}\right) ;\end{cases}
\end{aligned}
$$




$$
\begin{gathered}
\tilde{\pi}_{i}^{(k)}(g)=\sum_{u \in U} \delta_{g\left(J\left(u ; \theta_{0}\right)-J\left(u ; \theta_{1}\right)\right), i} \tilde{P}\left(u ; \theta_{k}\right), i \in\left(C_{-}, C_{+}\right) ; \\
\tilde{\pi}_{C_{+}(k)}^{(k)}(g)=\sum_{i \geq C_{+}} \sum_{u \in U} \delta_{g\left(J\left(u ; \theta_{0}\right)-J\left(u ; \theta_{1}\right)\right), i} \tilde{P}\left(u ; \theta_{k}\right), \\
\tilde{\pi}_{C_{-}}^{(k)}(g)=\sum_{i \leq C_{-}} \sum_{u \in U} \delta_{g\left(J\left(u ; \theta_{0}\right)-J\left(u ; \theta_{1}\right)\right), i} \tilde{P}\left(u ; \theta_{k}\right),
\end{gathered}
$$

$\tilde{R}^{(k)}(g), \tilde{Q}^{(k)}(g), \tilde{R}^{(k)}, \tilde{Q}^{(k)}$ are the sub-matrices of $\tilde{P}^{(k)}(g), \tilde{P}^{(k)}$ respectively, similar to $R^{(k)}$, $Q^{(k)}$. Denote the matrix $\hat{S}^{(k)}=\mathbf{I}_{N}-Q^{(k)}-\varepsilon\left(\tilde{Q}^{(k)}-Q^{(k)}\right), k \in\{0,1\}$.

Theorem 1. If the hypothetical model (1), (4) is distorted according to (7), (8), and $\left|S^{(k)}\right| \neq$ $0,\left|\hat{S}^{(k)}\right| \neq 0, k \in\{0,1\}$, then for the SPRT the conditional mathematical expectation of the sample size $\bar{t}^{(k)}$ and the factual values $\bar{\alpha}, \bar{\beta}$ of the error type I and II probabilities for the distorted model differ from the correspondent characteristics calculated for the hypothetical model, by the values of the order $\mathcal{O}(\varepsilon)$ :

$$
\begin{gathered}
\bar{t}^{(k)}-t^{(k)}=\varepsilon\left(\left(\tilde{\pi}^{(k)}-\pi^{(k)}\right)^{\prime}+\left(\pi^{(k)}\right)^{\prime}\left(S^{(k)}\right)^{-1}\left(\tilde{Q}^{(k)}-Q^{(k)}\right)\right) \times \\
\times\left(S^{(k)}\right)^{-1} \mathbf{1}_{N-2}+\mathcal{O}\left(\varepsilon^{2}\right) ; \\
\bar{\alpha}-\alpha=\varepsilon\left(( \pi ^ { ( 0 ) } ) ^ { \prime } \left(( S ^ { ( 0 ) } ) ^ { - 1 } \left(\left(\tilde{Q}^{(0)}-Q^{(0)}\right)\left(S^{(0)}\right)^{-1} R^{(0)}+\right.\right.\right. \\
\left.\left.\left.+\tilde{R}^{(0)}-R^{(0)}\right)\right)_{(2)}+\left(\tilde{\pi}^{(0)}-\pi^{(0)}\right)^{\prime} B_{(2)}^{(0)}+\tilde{\pi}_{C_{+}}^{(0)}-\pi_{C_{+}}^{(0)}\right)+\mathcal{O}\left(\varepsilon^{2}\right), \\
\bar{\beta}-\beta=\varepsilon\left(( \pi ^ { ( 1 ) } ) ^ { \prime } \left(( S ^ { ( 1 ) } ) ^ { - 1 } \left(\left(\tilde{Q}^{(1)}-Q^{(1)}\right)\left(S^{(1)}\right)^{-1} R^{(1)}+\right.\right.\right. \\
\left.\left.\left.+\tilde{R}^{(1)}-R^{(1)}\right)\right)_{(1)}+\left(\tilde{\pi}^{(1)}-\pi^{(1)}\right)^{\prime} B_{(1)}^{(1)}+\tilde{\pi}_{C_{-}}^{(1)}-\pi_{C_{-}}^{(1)}\right)+\mathcal{O}\left(\varepsilon^{2}\right) .
\end{gathered}
$$

Proof. The detailed proof is given in (Kharin 2013).

Using this theory, the performance characteristics can be calculated for tests from a family of sequential tests that makes possible to construct the robust sequential test by the minimax of the risk criterion.

In (Kharin and Kishylau 2015) the results are generalized to the case of arbitrary probability distribution of observations.

The case of inhomogeneous data - the model of time series with a trend - is analyzed in (Kharin and Tu 2017).

The case of Markov chains is considered in details in (Kharin 2013).

\section{Case of composite hypotheses}

\subsection{Mathematical model and notation}

Let on a probability space $(\Omega, \mathcal{F}, \mathcal{P})$ a random sequence of independent inhomogeneous variables $x_{1}, x_{2}, \ldots \in \mathbf{R}$ be observed, with p.d.f.s $p_{1}(x \mid \theta), p_{2}(x \mid \theta), \ldots$, where $\theta \in \Theta \subseteq \mathbf{R}^{k}$ is an unknown value of the random parameters vector. The prior probability density function $p(\theta)$ of this vector in the Bayesian setting is supposed to be known. There are two composite hypotheses on the value of $\theta$ :

$$
\mathcal{H}_{0}: \theta \in \Theta_{0}, \quad \mathcal{H}_{1}: \theta \in \Theta_{1} ; \quad \Theta_{0} \cup \Theta_{1}=\Theta, \Theta_{0} \cap \Theta_{1}=\emptyset .
$$

Introduce the notation:

$$
\mathbf{1}_{S}(s)= \begin{cases}1, & s \in S \\ 0, & s \notin S\end{cases}
$$




$$
W_{i}=\int_{\Theta_{i}} p(\theta) d \theta ; w_{i}(\theta)=\frac{1}{W_{i}} \cdot p(\theta) \cdot \mathbf{1}_{\Theta_{i}}(\theta), \theta \in \Theta, i=0,1
$$

Denote by

$$
\Lambda_{n}=\Lambda_{n}\left(x_{1}, \ldots, x_{n}\right)=\log \frac{\int_{\Theta} w_{1}(\theta) p_{1}\left(x_{1} \mid \theta\right) d \theta \cdots \int_{\Theta} w_{1}(\theta) p_{n}\left(x_{n} \mid \theta\right) d \theta}{\int_{\Theta} w_{0}(\theta) p_{1}\left(x_{1} \mid \theta\right) d \theta \cdots \int_{\Theta} w_{0}(\theta) p_{n}\left(x_{n} \mid \theta\right) d \theta}
$$

the logarithm of the generalized likelihood ratio statistic, that is calculated by $n$ observations $x_{1}, \ldots, x_{n}$.

To test the hypotheses (9), the following parametric family of Bayesian sequential tests is used:

$$
\begin{gathered}
N=\min \left\{n \in \mathbf{N}: \Lambda_{n} \notin\left(C_{-}, C_{+}\right)\right\}, \\
d=\mathbf{1}_{\left[C_{+},+\infty\right)}\left(\Lambda_{N}\right),
\end{gathered}
$$

where $N$ is the random number of the observation that determines the stopping time, after that observation the decision $d$ is made according to the decision rule (12). The decision $d=i$ means that the hypothesis $\mathcal{H}_{i}$ is accepted, $i=0,1 ; C_{-}<0, C_{+}>0$ are parameters of the test (11), (12):

$$
C_{-}=\ln \left(\beta_{0} /\left(1-\alpha_{0}\right)\right), C_{+}=\ln \left(\left(1-\beta_{0}\right) / \alpha_{0}\right),
$$

where $\alpha_{0}, \beta_{0} \in\left(0, \frac{1}{2}\right)$ are some values close to maximal admissible levels of error type I and II probabilities (Wald 1947). The actual values $\alpha, \beta$ of the error type I and II probabilities may deviate from $\alpha_{0}, \beta_{0}$.

For calculation of $\alpha, \beta$ and mathematical expectations of the random variable $N$ determined by (11), let us use a stochastic approximation of the statistic $\Lambda_{n}, n \in \mathbf{N}$. Let $m \in \mathbf{N}$ be a parameter of the approximation, $h=\left(C_{+}-C_{-}\right) / m$. Let $p_{\Lambda_{n}}(u)$ be the probability density function of the statistic (10); $p_{\Lambda_{n+1} \mid \Lambda_{n}}(u \mid y)$ be the conditional probability density function, $n \in \mathbf{N}$; let $R^{(n)}(\theta)$ and $Q^{(n)}(\theta)$ be the blocks of the sizes $m \times 2$ and $m \times m$ respectively for the approximating Markov chain, $\mathbf{I}_{k}$ is the identity matrix of the size $k, \mathbf{0}_{(2 \times m)}$ is the matrix of the size $(2 \times m)$, with all elements equal to 0 . Let $\pi(\theta)=\left(\pi_{i}(\theta)\right)$ be the vector of initial probabilities of the states $1, \ldots, m$ for the approximating random sequence; $\pi_{0}(\theta), \pi_{m+1}(\theta)$ be the initial probabilities of the absorbing states 0 and $m+1 ; \mathbf{1}_{m}$ be the vecor of size $m$, with all components equal to 1 . Denote:

$$
\begin{gathered}
S(\theta)=\mathbf{I}_{m}+\sum_{i=1}^{\infty} \prod_{j=1}^{i} Q^{(j)}(\theta) \\
B(\theta)=R^{(1)}(\theta)+\sum_{i=1}^{\infty} \prod_{j=1}^{i} Q^{(j)}(\theta) R^{(i+1)}(\theta) .
\end{gathered}
$$

Let $B_{(j)}(\theta)$ be the column number $j$ of the matrix $B(\theta), j=1,2 ; t_{i}=\mathrm{E}\left\{N \mid \theta \in \Theta_{i}\right\}, i=0,1$; $t=\mathrm{E}\{N\}$.

\subsection{Distortions}

Let the hypothetical model described above be distorted, although the Bayesian sequential test (11), (12) is used. The test is constructed on the basis of the hypothetical probability density functions $p(\theta), p_{n}\left(x_{1}, \ldots, x_{n} \mid \theta\right)$, but these probability density functions are simultaneously distorted. Actually, the parameters vector $\theta$ has the distorted probability density function

$$
\bar{p}(\theta)=\left(1-\varepsilon_{\theta}\right) \cdot p(\theta)+\varepsilon_{\theta} \cdot \tilde{p}(\theta), \theta \in \Theta,
$$

where $\varepsilon_{\theta} \in\left[0, \frac{1}{2}\right)$ is the probability of "contamination" w.r.t. the probability density of $\theta$, and $\tilde{p}(\theta)$ is a "contaminating" probability density function that differs from $p(\theta)$. The distorted 
conditional probability density function of observations is also a mixture of the hypothetical $p_{n}(\cdot \mid \cdot)$ and the "contaminating" $\tilde{p}_{n}(\cdot \mid \cdot)$ probability density functions:

$$
\begin{aligned}
\bar{p}_{n}\left(x_{1}, \ldots, x_{n} \mid \theta\right)= & \left(1-\varepsilon_{x}\right) \cdot p_{n}\left(x_{1}, \ldots, x_{n} \mid \theta\right)+\varepsilon_{x} \cdot \tilde{p}_{n}\left(x_{1}, \ldots, x_{n} \mid \theta\right), \\
& \theta \in \Theta, x_{1}, \ldots, x_{n} \in \mathbf{R}, n \in \mathbf{N},
\end{aligned}
$$

where $\varepsilon_{x} \in\left[0, \frac{1}{2}\right)$ can be interpreted as the probability of an "outlier" presence (see Huber and Ronchetti (2009)) w.r.t. the observations $x_{1}, x_{2}, \ldots$

Let $\tilde{\pi}(\theta), \tilde{\pi}_{0}(\theta), \tilde{\pi}_{m+1}(\theta), \tilde{Q}^{(n)}(\theta), \tilde{R}^{(n)}(\theta)$ be the elements calculated analogously to $\pi(\theta)$, $\pi_{0}(\theta), \pi_{m+1}(\theta), Q^{(n)}(\theta), R^{(n)}(\theta)$ by replacing the hypothetical p.d.f. $p_{n}\left(x_{1}, \ldots, x_{n} \mid \theta\right)$ with the "contaminating" p.d.f. $\tilde{p}_{n}\left(x_{1}, \ldots, x_{n} \mid \theta\right)$ in the probability distribution of the approximating random sequence; $\Delta \pi_{0}(\theta)=\tilde{\pi}_{0}(\theta)-\pi_{0}(\theta), \Delta \pi_{1}(\theta)=\tilde{\pi}_{m+1}(\theta)-\pi_{m+1}(\theta) ; \bar{t}(\theta)$ and $\bar{\gamma}_{\mathcal{H}_{i}}(\theta)$, $i=0,1$, be the conditional mathematical expectation of the sample size and the conditional probability of acceptance of the hypothesis $\mathcal{H}_{i}$ respectively, provided the parameters vecor value is $\theta$, for the distorted model (13), (14).

\subsection{Robustness analysis via asymptotic expansions of the performance char- acteristics}

Introduce the notation:

$$
\begin{gathered}
\tilde{W}_{i}=\int_{\Theta_{i}} \tilde{p}(\theta) d \theta \\
A(\theta)=\left((\tilde{\pi}(\theta)-\pi(\theta))^{\prime} S(\theta)+\right. \\
\left.(\pi(\theta))^{\prime} \cdot \sum_{i=1}^{\infty} \sum_{j=1}^{i} \prod_{k=1}^{j-1} Q^{(k)}(\theta)\left(\tilde{Q}^{(j)}(\theta)-Q^{(j)}(\theta)\right) \prod_{k=j+1}^{i} Q^{(k)}(\theta)\right) \cdot \mathbf{1}_{m} ; \\
F_{i}(\theta)=\Delta \pi_{i}(\theta)+(\tilde{\pi}(\theta)-\pi(\theta))^{\prime} B_{(i+1)}(\theta)+\tilde{R}^{(1)}(\theta)-R^{(1)}(\theta)+ \\
\sum_{l=1}^{\infty}\left(\sum_{j=1}^{l} \prod_{k=1}^{j-1} Q^{(k)}\left(\tilde{Q}^{(j)}(\theta)-Q^{(j)}(\theta)\right) \prod_{k=j+1}^{l} Q^{(k)}(\theta) R^{(l+1)}(\theta)+\right. \\
\left.\prod_{j=1}^{l} Q^{(j)}(\theta)\left(\tilde{R}^{(l+1)}(\theta)-R^{(l+1)}(\theta)\right)\right), i=0,1 .
\end{gathered}
$$

Theorem 2. Let the random sequence (10) satisfies the Markov property, $\forall \theta \in \Theta$, the probability density functions $p_{\Lambda_{1}}(u), p_{\Lambda_{n+1} \mid \Lambda_{n}}(u \mid y)$ be differentiable functions w.r.t. the variable $u \in\left[C_{-}, C_{+}\right]$, and $\exists C \in(0,+\infty)$ :

$$
\left|\frac{d p_{\Lambda_{1}}(u)}{d u}\right| \leq C,\left|\frac{d p_{\Lambda_{n+1} \mid \Lambda_{n}}(u \mid y)}{d u}\right| \leq C, u, y \in\left[C_{-}, C_{+}\right], n \in \mathbf{N} .
$$

Then under simultaneous distortions (13), (14) the following asymptotic expansions hold for the error type $I$ and II probabilities $\bar{\alpha}, \bar{\beta}$ at $\varepsilon_{\theta} \rightarrow 0, \varepsilon_{x} \rightarrow 0, h \rightarrow 0$ :

$$
\begin{gathered}
\bar{\alpha}=\alpha+\varepsilon_{x} \cdot \frac{1}{W_{0}} \cdot \int_{\Theta_{0}} F_{1}(\theta) p(\theta) d \theta+ \\
\varepsilon_{\theta}\left(\frac{1}{W_{0}^{2}} \cdot \int_{\Theta_{0}}(\tilde{p}(\theta)-p(\theta)) d \theta \cdot \int_{\Theta_{0}} \gamma_{\mathcal{H}_{1}}(\theta) p(\theta) d \theta+\frac{1}{W_{0}} \cdot \int_{\Theta_{0}} \gamma_{\mathcal{H}_{1}}(\theta)(\tilde{p}(\theta)-p(\theta)) d \theta\right)+ \\
\mathcal{O}\left(\varepsilon_{x}^{2}\right)+\mathcal{O}\left(\varepsilon_{\theta}^{2}\right)+\mathcal{O}(h) ; \\
\bar{\beta}=\beta+\varepsilon_{x} \cdot \frac{1}{W_{1}} \cdot \int_{\Theta_{1}} F_{0}(\theta) p(\theta) d \theta+ \\
\varepsilon_{\theta}\left(\frac{1}{W_{1}^{2}} \cdot \int_{\Theta_{1}}(\tilde{p}(\theta)-p(\theta)) d \theta \cdot \int_{\Theta_{1}} \gamma_{\mathcal{H}_{0}}(\theta) p(\theta) d \theta+\frac{1}{W_{1}} \cdot \int_{\Theta_{1}} \gamma_{\mathcal{H}_{0}}(\theta)(\tilde{p}(\theta)-p(\theta)) d \theta\right)+ \\
\mathcal{O}\left(\varepsilon_{x}^{2}\right)+\mathcal{O}\left(\varepsilon_{\theta}^{2}\right)+\mathcal{O}(h) .
\end{gathered}
$$


Proof. The detailed proof is given in (Kharin 2013).

Denote by $\bar{t}_{i}, i=0,1$, the conditional mathematical expectation of the random number of observations $N$ provided the hypothesis $\mathcal{H}_{i}$ is true, if the hypothetical model is distorted according to (13), (14).

Theorem 3. Under the conditions of Theorem 2, the conditional expected sample sizes satisfy the asymptotic expansions:

$$
\begin{gathered}
\bar{t}_{i}=t_{i}+\varepsilon_{x} \cdot \frac{1}{W_{i}} \cdot \int_{\Theta_{i}} A(\theta) p(\theta) d \theta+\varepsilon_{\theta} \cdot\left(t_{i} \cdot\left(\tilde{W}_{i}-W_{i}\right)+\frac{1}{W_{i}} \cdot \int_{\Theta_{i}} t(\theta)(\tilde{p}(\theta)-p(\theta)) d \theta\right)+ \\
\mathcal{O}\left(\varepsilon_{x}^{2}\right)+\mathcal{O}\left(\varepsilon_{\theta}^{2}\right)+\mathcal{O}(h), i=0,1 .
\end{gathered}
$$

Theorem 4. If the conditions of Theorem 2 are satisfied, then the following asymptotic expansion holds for the expected sample size:

$$
\bar{t}=t+\varepsilon_{x} \cdot \int_{\Theta} A(\theta) p(\theta) d \theta+\varepsilon_{\theta} \cdot \int_{\Theta} t(\theta)(\tilde{p}(\theta)-p(\theta)) d \theta+\mathcal{O}\left(\varepsilon_{x}^{2}\right)+\mathcal{O}(h) .
$$

Proof. Proofs of Theorems 3, 4 are presented in Kharin (2013).

This theory is used to calculate the performance characteristics of the sequential tests under distortions and to construct the robust sequential test (Kharin 2017). The approach is presented in (Kharin 2016) with some numerical results.

\section{Conclusion}

The problem of robustness analysis for sequential statistical decision rules is considered in the paper. The cases of simple and composite hypotheses are analyzed. Asymptotic expansions are constructed for the performance characteristics of the sequential statistical decision rules under distortion. Analyzing the constructed expansions and constructing the similar for sequential test from a generalized families, robust sequential statistical decision rules can be constructed. The results are also applied for the decision making in case of many hypotheses (Ton and Kharin 2019).

\section{References}

Aivazian S (1959). "Comparison of Optimal Properties of Neyman-Pearson and Wald Tests." Probability Theory and its Applications, 4(1), 86-93.

Galinskij V, Kharin A (1999). "On Minimax Robustness of Bayesian Statistical Prediction." Probability Theory and Mathematical Statistics, pp. 259-266.

Ghosh B, Sen P (1991). Handbook of Sequential Analysis. Marcel Dekker, New York, Basel, Hong Kong.

Huber P, Ronchetti E (2009). Robust Statistics. Wiley, New York.

Jennison C, Turnbull B (2000). Group Sequential Methods with Applications to Clinical Trials. Chapman \& Hall / CRC, Boca Raton.

Kharin A (2005). "Robust Bayesian Prediction under Distortions of Prior and Conditional Distributions." Journal of Mathematical Sciences, 126(1), 992-997.

Kharin A (2013). Robustness in Bayesian and Sequential Statistical Decision Rules. Belarusian State University, Minsk. ISBN 978-9855188750. 
Kharin A (2016). "Performance and Robustness Evaluation in Sequential Hypotheses Testing." Communications in Statistics - Theory and Methods, 45(6), 1693-1709.

Kharin A (2017). "An Approach to Asymptotic Robustness Analysis of Sequential Tests for Composite Parametric Hypotheses." Journal of Mathematical Sciences (United States), 227(2), 196-203.

Kharin A, Kishylau D (2015). "Robust Sequential Test for Hypotheses about Discrete Distributions in the Presence of "Outliers"." Journal of Mathematical Sciences (United States), 205(1), 68-73.

Kharin A, Tu T (2017). "Performance and Robustness Analysis of Sequential Hypotheses Testing for Time Series with Trend." Austrian Journal of Statistics, 46(3-4), 23-36.

Kharin Y (1997). "Robustness of Clustering under Outliers." Lecture Notes in Computer Science (including subseries Lecture Notes in Artificial Intelligence and Lecture Notes in Bioinformatics), 1280, 501-511.

Kharin Y (2011). "Robustness of the Mean Square Risk in Forecasting of Regression Time Series." Communications in Statistics - Theory and Methods, 40(16), 2893-2906.

Kharin Y, Vecherko E (2013). "Statistical Estimation of Parameters for Binary Markov Chain Models with Embeddings." Discrete Mathematics and Applications, 23(2), 153-169.

Kharin Y, Zhuk E (1998). "Filtering of Multivariate Samples Containing "Outliers" for Clustering." Pattern Recognition Letters, 19(12), 1077-1085.

Lai T (2001). "Sequential Analysis: Some Classical Problems and New Challenges." Statistica Sinica, 11, 303-408.

Maevskii V, Kharin Y (2002). "Robust Regressive Forecasting under Functional Distortions in a Model." Automation and Remote Control, 63(11), 1803-1820.

Pandit P, Gudaganavar N (2009). "On Robustness of a Sequential Test for Scale Parameter of Gamma and Exponential Distributions." Applied Mathematics, 1, 274-278.

Quang P (1985). "Robust Sequential Testing." Annals of Statistics, 13(2), 638-649.

Ton T, Kharin A (2019). "Sequential Probability Ratio Test for Many Simple Hypotheses on Parameters of Time Series with Trend." Journal of the Belarusian State University. Mathematics and Informatics, pp. 35-46.

Wald A (1947). Sequential Analysis. John Wiley and Sons, New York.

\section{Affiliation:}

Alexey Kharin

Department of Probability Theory and Mathematical Statistics

Belarusian State University

Independence av. 4, Minsk 220030, Belarus

E-mail: KharinAY@bsu.by

URL: https : //fpmi . bsu . by/?guid=32921

\section{Austrian Journal of Statistics}

published by the Austrian Society of Statistics

Volume 49

April 2020 http://www.ajs.or.at/

http://www.osg.or.at/

Submitted: 2019-12-15

Accepted: 2020-03-03 